\title{
GLAD!
}

Revue sur le langage, le genre, les sexualités

\section{Maude Vadot, Françoise Roche \& Chahrazed Dahou (dir.). 2017. Genre et sciences du langage : enjeux et perspectives}

Noémie Marignier

\section{(2) OpenEdition}

Journals

Édition électronique

URL : http://journals.openedition.org/glad/790

DOI : $10.4000 /$ glad. 790

ISSN : 2551-0819

Éditeur

Association GSL

\section{Référence électronique}

Noémie Marignier, « Maude Vadot, Françoise Roche \& Chahrazed Dahou (dir.). 2017. Genre et sciences du langage : enjeux et perspectives », GLAD! [En ligne], 03 | 2017, mis en ligne le 10 décembre 2017, consulté le 20 janvier 2021. URL : http://journals.openedition.org/glad/790 ; DOI : https://doi.org/ $10.4000 /$ glad.790

Ce document a été généré automatiquement le 20 janvier 2021.

\section{(c) (i) (9)}

La revue GLAD! est mise à disposition selon les termes de la Licence Creative Commons Attribution Pas d'Utilisation Commerciale - Pas de Modification 4.0 International. 


\title{
Maude Vadot, Françoise Roche \& Chahrazed Dahou (dir.). 2017. Genre et sciences du langage : enjeux et perspectives
}

\author{
Noémie Marignier
}

\section{RÉFÉRENCE}

Maude Vadot, Françoise Roche \& Chahrazed Dahou (dir.). 2017. Genre et sciences $d u$ langage : enjeux et perspectives. Montpellier : Presses Universitaires de la Méditerranée.

1 L'ouvrage Genre et sciences du langage : enjeux et perspectives coordonné par Maude Vadot, Françoise Roche et Chahrazed Dahou, paru au printemps 2017 aux Presses universitaires de la Méditerranée, se présente comme les actes du colloque consacré à ces questions, colloque qui a eu lieu en novembre 2014 à l'université Paul Valéry Montpellier 3.

2 L'ouvrage fait la part belle à la jeune recherche, puisque neuf des treize chapitres qui le composent sont écrits par des jeunes chercheur-ses (doctorant-es ou docteur-es). Il entend ainsi présenter les perspectives variées et actuelles des recherches linguistiques sur le genre, aussi bien en didactique qu'en analyse du discours et sociolinguistique, en grammaire, etc. Mais c'est également le concept de genre qui est traité de manière diverse : les coordinatrices notent qu'elles n'ont pas tranché entre une utilisation au singulier ou au pluriel de genre(s), ni entre l'utilisation de la notion comme simple variable ou comme catégorie d'analyse critique. S'entrecroisent donc des perspectives différentes à la fois en ce qui concerne le genre, et en ce qui concerne les disciplines des sciences du langage, même si l'on remarque que c'est la définition de genre comme système de bicatégorisation hiérarchisée des individus qui est la plus présente dans les chapitres de l'ouvrage. 
3 Celui-ci est divisé en trois parties : "Épistémologie et histoire des études de genre ", "Genre et étude des pratiques langagières", «Genre et didactique des langues vivantes"; il s'achève par une conclusion d'Anne-Marie Houdebine consacrée à l'histoire du féminisme en sciences du langage.

4 La première partie est la plus théorique et est consacrée à des questions épistémologiques. Véronique Perry et Lucy Michel s'intéressent à la manière dont le genre grammatical est conceptualisé par les linguistes, et montrent que la force idéologique du genre est un nœud central pour comprendre ses manifestations en langue. Maria Candea fournit une réflexion épistémologique sur les questions de perception et de stéréotypes de genre dans les dispositifs expérimentaux en phonétique et sociolinguistique. Andrea d'Urso propose d'articuler deux traditions théoriques matérialistes différentes en ce qui concerne la sémiologie et le genre.

5 Le chapitre de Véronique Perry est consacré à l'héritage constructiviste oublié d'Edward Sapir. Celui-ci permet des réflexions intéressantes sur le genre: Sapir a notamment montré que le genre est un concept qui n'appartient pas au domaine strictement linguistique. Il faut plutôt considérer que le genre est une métaphore du binaire qui se transpose dans les catégories de langue, et le genre en langue est alors moins un fait linguistique qu'un "artifice idéologique» (p. 32). Envisager les langues comme « des représentations du monde culturellement marquées et normées » permet alors, selon Perry, de reconceptualiser la bicatégorisation en langue, sans tomber dans une essentialisation du genre : cela permet de penser de manière plus souple le lien entre genre et langue.

6 L'article de Lucy Michel «Le "masculin culturel", un pied de nez au "masculin générique" ?" résonne de manière intéressante avec l'article de Perry, en ce qu'il fournit une démonstration sémantique et grammaticale du genre considéré comme production idéologique qui viendrait s'inscrire en langue. Michel offre en effet une réflexion sur la notion de généricité en ce qui concerne le genre grammatical. Après un utile rappel de la distinction entre emploi générique et valeur générique, Michel montre à partir d'une étude de corpus d'entrées du dictionnaire qu'il y a tout lieu de douter de l'effectivité de cette valeur générique du masculin. C'est une approche prototypique du genre grammatical que propose l'auteure : loin d'être simplement un indicateur référentiel, le genre grammatical porte des représentations socioculturelles, souvent stéréotypées, de ce que sont le masculin et le féminin. Cette approche par la sémantique du genre grammatical permet d'éviter les écueils essentialistes d'une certaine approche référentialiste du genre grammatical et de congédier au niveau de l'analyse linguistique même la pertinence d'une valeur générique du masculin.

7 L'article de Maria Candea, «Qu'est-ce que le genre apporte aux études sur la perception de la parole ?» propose une réflexion sur un aspect rarement étudié en linguistique, dans le domaine de la perception. Candea présente tout d'abord des recherches antérieures, notamment des enquêtes expérimentales. Celles-ci montrent que l'identification préalable des locuteur-ices par des caractéristiques sociales/raciales/ sexuées activent chez les personnes qui perçoivent ces extraits (les « juges») un certain nombre de stéréotypes sur ces locuteur-ices. Mais les juges peuvent déjouer cette activation de stéréotypes notamment lorsqu'ils ont conscience "de l'utilisation qui peut être faite de leurs réponses » (p. 86). Candea invite alors les études de perception à s'emparer de ces questions encore marginales et à complexifier leurs dispositifs d'enquêtes, aussi bien par la diversité et la non-binarité des locuteurs dont la parole est 
l'objet du test, mais aussi par la prise en compte des affects, stéréotypes, croyances produites par les juges.

8 L'article d'Andrea d'Urso s'appuie sur deux ancrages théoriques différents: le féminisme matérialiste (notamment Silvia Federici) et la philosophie et sémiologie matérialistes de Ferruccio Rossi-Landi. Selon ce dernier, le langage est un travail et un marché, c'est-à-dire qu'il y a un travail de production des signes linguistiques qui s'articule à la création d'une plus-value idéologique et linguistique, au bénéfice d'une minorité dominante. Selon d'Urso, il est possible de croiser ces analyses avec des analyses féministes matérialistes qui ont montré qu'existait une plus-value créée par le travail des femmes, quand bien même celles-ci ne sont pas salariées, et qu'une " exploitation non monétaire des femmes rend possible une accumulation monétaire " (p. 52). C'est à croiser ces perspectives autour d'une « extension du concept de plusvalue » qu'appelle d'Urso.

9 Cette partie s'avère tout à fait intéressante dans la mesure où elle propose effectivement des perspectives théoriques novatrices en ce qui concerne les recherches linguistiques sur le genre, que ce soit dans le renouvellement de questions anciennes les liens entre genre «social » et grammatical (Perry), entre sens, référence et marque du genre (Michel) -, dans la remise en question de certaines méthodologies des sciences du langage à la lumière du genre (Candea), ou encore dans la proposition de nouvelles articulations théoriques (d'Urso).

10 La deuxième partie de l'ouvrage, "Genre et étude des pratiques langagières ", est consacrée à l'étude de productions discursives diverses. On peut d'ailleurs saluer la diversité des corpus sur lesquels portent les contributions: éloges funèbres des Ursulines du XVII ${ }^{e}$ au XIXe siècle pour Sylvie Dubois et Natacha Jeudy, professions de foi des députées en France pour Magali Guaresi, forums de discussion consacrés aux transitions FtM pour Alice Coutant, tweets et hashtags mobilisant des stéréotypes de genre pour Camille Lagarde-Belleville et Michel Otell. Ces chapitres viennent présenter différentes manières de mettre le genre en discours, à différents niveaux.

11 Jeudy et Dubois présentent le genre de l'éloge funèbre des religieuses dans son contexte social et culturel, s'attachant à la circulation des éloges aussi bien qu'aux thèmes qu'ils présentent et à leurs structures argumentatives, dans leurs diversités. Elles montrent particulièrement leurs différentes fonctions au sein des communautés de religieuses ; aux fonctions informatives et édificatrices s'ajoutent des fonctions de création de liens d'échanges entre les monastères entre lesquels ils circulent, mais également de diffusion de valeurs et de représentation.

12 L'article de Coutant s'intéresse aux liens effectués par les locuteurs FtM sur les forums entre genre grammatical et présentation de soi : elle examine les difficultés pour ces locuteurs à négocier la question de l'utilisation du genre grammatical, qui indexe leur identité ressentie, mais peut parfois se trouver en décalage avec leur identité perçue. Cela conduit certains locuteurs à la production de stratégies langagières en ce qui concerne les marques du genre - notamment ce que les locuteurs nomment « parler au neutre ». Il s'agit ainsi pour Coutant de s'intéresser à la manière dont ces locuteurs mettent en discours leurs stratégies d'utilisation du genre grammatical.

13 Ce sont d'autres manifestations du genre dans le langage qui intéressent Guaresi : à partir d'une analyse logométrique doublée d'une analyse qualitative, celle-ci montre que les candidates aux élections législatives de 1997, dans leurs professions de foi, mobilisent de manière beaucoup plus saillante que les hommes le pronom je (les 
candidats préférant le nous). Les candidates produisent par là des énoncés qui mettent l'accent sur leur identité personnelle en se conformant à des représentations stéréotypées des qualités féminines. Pour Guaresi, cela obéit à des «modalités de légitimation genrées ", c'est-à-dire que les candidates produisent des éthos obéissant aux contraintes de l'arène électorale, celle-ci valorisant des représentations traditionnelles de la féminité.

14 C'est également la question des stéréotypes qui intéresse Lagarde-Belleville et Otell autour des tweets comportant les hashtags \#tweetcommeunefille et \#tweetcommeunmec. Ces hashtags sont utilisés par les internautes pour stéréotyper de manière ludique les discours féminins et masculins. Pour autant, il ne s'agit pas selon les auteures d'une démarche critique: ces tweets contribuent à renforcer les représentations stéréotypées de genre, la dichotomie entre les sexes. De plus les stéréotypes associés au féminin sont dans leur grande majorité dépréciatifs, tandis que les stéréotypes associés au masculin sont plus variés, et ce, quel que soit le genre des producteur-ices des tweets.

15 Ces chapitres examinent la pression qui peut s'exercer sur les locuteur-ices au niveau de leurs pratiques langagières afin de produire des représentations de genre conformes à ce qui est attendu - que ce soit dans la manière de se catégoriser (Coutant) ou de catégoriser autrui, ou dans les formes langagières utilisées plus généralement. Ces articles montrent bien comment les discours s'appuient sur l'impératif de dichotomie entre les sexes, tout cela s'effectuant au détriment des femmes (Guaresi, LagardeBelleville) par les stéréotypes qui sont convoqués, mais aussi reproduits.

La troisième partie de l'ouvrage, "Genre et didactique des langues vivantes", se caractérise par sa très grande cohérence thématique. Ces articles s'intéressent aussi bien aux représentations de genre supposées chez les publics d'apprenant-es qu'à celles véhiculées par les outils de formation en langue française.

17 Le chapitre d'Émilie Lebreton s'appuie sur une enquête de terrain réalisée lors de formations en langue française proposées à un public de femmes et hommes migrant-es en France ; elle analyse les réactions et les attentes exprimées par ceulles-ci, ainsi que celles des formateur-ices. La contribution s'articule à la question de la volonté, chez les femmes migrantes, d'un apprentissage rigoureux et normé de la langue française. Lebreton vient nuancer cette affirmation : d'une part, cette demande est partagée par les hommes, d'autre part elle n'est pas une volonté de norme pour la norme, mais s'inscrit aussi dans la construction d'un parcours migratoire, d'une identité d'apprenante et d'un parcours professionnel dans lequel les femmes ne seraient pas passives.

18 Maude Vadot s'intéresse quant à elle au contenu des manuels - donc aux supports de formation - destinés aux publics de migrants, en s'interrogeant sur les notions de genre qui doivent, selon les autorités dédiées, être transmises aux migrants. Vadot met en contraste le référentiel gouvernemental «français langue d'intégration » et les manuels dédiés à la formation en français en ce qui concerne les représentations du genre. Si le premier insiste particulièrement sur les valeurs d'égalité femme-homme qui doivent être transmises aux migrants lors de leur formation - laissant entendre qu'ils s'adressent à un public forcément sexiste, les manuels ne sont pas forcément exemplaires sur ces questions: les représentations différenciées et hiérarchisées des hommes et des femmes y sont bien présentes. 
19 Grâce Ranchon s'intéresse également aux manuels mais dans un autre contexte : celui de l'enseignement $d u$ français dans le monde. Sa contribution porte plus particulièrement sur l'hétéronormativité. Elle observe deux orientations principales en ce qui concerne les représentations du couple et de la sexualité : d'une part le manuel le plus progressiste (évoquant la variété des modèles familiaux français) n'accorde que très peu de place aux questions de genre, de sexualité ou de conjugalité ; d'autre part, dans les manuels où la question est abordée, les représentations normées ont la part belle.

20 Les contributions à cette partie s'avèrent particulièrement complémentaires de par leurs points de vue et leurs choix de corpus: enquête auprès des personnes apprenantes et de leur formateur-ices (Lebreton), analyse de manuels quantitative (Vadot) ou qualitative (Ranchon), étude de l'apprentissage destiné aux migrant-es (Lebreton, Vadot) ou destiné à des publics hors de France (Ranchon), analyse des représentations du genre supposées chez les migrant-es (Vadot) mais aussi des représentations du genre chez les rédacteur-ices de manuels (Ranchon).

21 Enfin, le chapitre de Mireille Baurens qui conclut cette partie offre un retour sur son parcours d'enseignante-chercheuse en didactique et genre: Baurens y présente notamment des stratégies pédagogiques pour amener les étudiant-es à interroger les questions de genre et à s'en saisir dans leurs pratiques et dans la construction de leurs savoirs, dans une dynamique collective et active.

22 Le chapitre conclusif d'Anne-Marie Houdebine offre une utile perspective historique sur les recherches sur le genre en sciences du langage en les replaçant dans le cadre plus large des combats et recherches féministes depuis les années 1960. Elle revient plus particulièrement sur la commission de féminisation des titres et des fonctions créée par Yvette Roudy en 1983, ainsi que sur les obstacles que la commission a dû affronter. On peut néanmoins regretter une conclusion un peu réductrice dans sa lecture « civilisationnelle » des discriminations envers les femmes. Il semble également dommage qu'un ouvrage composé de travaux de la jeune recherche se termine par la critique d'une nouvelle génération "conquise par les études de genre » qui abandonnerait le féminisme et qui prônerait avec "rigidité voire dogmatisme" «l'absence de différence et la mobilité sexuelle » (p. 299).

23 L'ouvrage nous semble au contraire présenter une multiplicité de recherches féministes sur le langage - ou en tout cas menées depuis la perspective critique du concept de genre - qui renouvellent les approches traditionnelles sans les désavouer.

24 En effet, l'ouvrage présente dans la diversité des articles les multiples manières dont le genre investit les discours, la langue, les représentations ; l'importance des stéréotypes genrés et leur fabrication complexe dans les pratiques langagières est mise en lumière. Si le genre est pour la plupart des auteur-es considéré comme un système de bicatégorisation, une idéologie hiérarchisante, cela ne donne pas lieu à une uniformisation des groupes sociaux et laisse la place à des questionnements plus actuels en sciences du langage, en ce qui concerne des thèmes comme la sexualité (Ranchon), les publics enquêtés (Coutant) ou les perspectives méthodologiques adoptées (Candea). Cette perspective non essentialiste permet de fines démonstrations, par exemple sur la construction d'éthos genrés entre stratégies discursives et reproductions de normes (Guaresi) ou sur le sémantisme du genre qui ne saurait se résoudre à l'opposition mâle/femelle (Michel). C'est bien la question des stratégies, des contraintes, des normes, dont les acteur-ices se saisissent que ce soit pour les endosser, 
les imposer ou encore les déjouer, qui est au cœur de l'ouvrage, et cela sans annuler une lecture du genre en termes de rapports de pouvoir.

INDEX

Mots-clés : genre, linguistique

Keywords : gender, linguistics

Thèmes : Actualités

\section{AUTEURS}

\section{NOÉMIE MARIGNIER}

Noémie Marignier est docteure en Sciences du Langage et Attachée Temporaire d'Enseignement et de Recherche à l'université Paris 3 Sorbonne Nouvelle. Ses travaux de recherche portent sur l'articulation entre corps/sexe/sexualité et discours. Elle a soutenu en 2016 une thèse en analyse du discours intitulée Les matérialités discursives du sexe. La construction et la déstabilisation des évidences du genre dans les discours sur les sexes atypiques. Elle est membre associée du laboratoire Clesthia (Université Paris 3 Sorbonne Nouvelle) ainsi que de Pléiade (Université Paris 13 Sorbonne Cité). 